\title{
Forecasts of Opportunity Opening Windows of Skill, Subseasonal and Beyond
}

Adapted from "Windows of Opportunity for Skillful Forecasts

Subseasonal to Seasonal and Beyond" by Annarita Mariotti (NOAA), Cory Baggett, Elizabeth

A. Barnes, Emily Becker, Amy Butler, Dan C. Collins, Paul A. Dirmeyer, Laura Ferranti, Nathaniel C. Johnson, Jeanine Jones, Ben P. Kirtman, Andrea L. Lang, Andrea Molod, Matthew Newman, Andrew W. Robertson, Siegfried Schubert, Duane E. Waliser, and John Albers. Published online in BAMS, May 2020. For the full, citable article, see DOI:10.1175 /BAMS-D-18-0326.1.
$\Lambda$ cross the globe, people desire forecasts out to several weeks with the skill of a 5-day weather forecast. A National Academies of Sciences, Engineering, and Medicine report suggests that subseasonal to seasonal (S2S) forecasts would, in 10 years, be used like weather forecasts are today. Research indicates that skillful S2S and seasonal to decadal (S2D) forecasts are possible beyond the predictable weather range of 14 days based on the existence of particular initial or climate conditions, but fluctuations in skill increase as the forecast range increases. As such, intermittent S2S/S2D skill can be exploited by strategically targeting windows of opportunity. The idea has analogies in weather: in some situations, winter forecasts tend to have more skill than summer forecasts. If we can identify what leads to predictable conditions with, say, forecast ensembles, we can potentially provide more useful S2S and S2D forecasts.

\section{Roots of forecast opportunities}

Reduced forecast skill is generally associated with atmospheric instabilities and weather regime transitions, especially at longer ranges. Quasistationary Rossby waves during boreal summer can also condition the skill of subseasonal prediction of extremes including heat waves, flooding, and short-term droughts. These waves often manifest as nearly circumglobal teleconnections, and there is mounting evidence that they can be amplified or sustained by tropical processes, land forcing, or even internal atmospheric resonance, possibly 
increasing the predictability of extremes such as heat waves well beyond 2 weeks. Taking full advantage of such enhanced predictability requires better understanding and simulation of the growth and persistence of associated processes, and the role of underpinning climate phenomena. For example, the potential for S2S predictability from tropical sources lies in the "slow," predictable evolution of large-scale tropical convection patterns, the 1-2 weeks for the extratropics to respond fully to tropical convective heating, and the several weeks for stratospheric-tropospheric interactions to occur.

An active Madden-Julian Oscillation (MJO) is a major tropical source of subseasonal predictability, in the tropics but also elsewhere. The large-scale convection of the MJO propagates eastward over 30-60 days, generating Rossby waves and an extratropical response 1-2 weeks later. Current dynamical models now exhibit significant MJO skill beyond 30 days.

Sudden stratospheric warming (SSW) events, associated with the weakening or reversal of polar vortex circulation, affect cyclone track frequency and surface air temperature anomalies for at least a month after they occur. Using January-March data for 1980-2015: (left) Daily 2-m temperature anomalies (K) within 30 days after an SSW event. (right) Those anomalies not within $\mathbf{3 0}$ days after an SSW event. *
An active El Niño-Southern Oscillation (ENSO)'s effects-and predictability influencesare felt on S2S to multiannual time scales. With some skill forecasting ENSO events at least a year in advance, we are still far from taking full advantage of ENSO-related predictability.

The stratosphere is also a source of S2S predictability. The quasibiennial oscillation (QBO) can modulate the predictability of the wintertime MJO as well as ENSO teleconnections. When the QBO and MJO are both active, forecasts of S2S extremes improve. Similarly, the winter stratospheric polar vortex also provides windows of enhanced S2S predictability. When the Northern Hemisphere polar vortex is displaced or split, the slow descent of anomalous winds and temperatures to the tropopause influences the jet stream and underlying weather for weeks to months. Likewise, southern stratospheric polar vortex variations affect Australian hot and dry extremes. Deterministic predictability of these stratospheric events is limited to 10-20 days, but ties to the state of MJO, QBO, and ENSO, among other climate factors, potentially allow increased surface predictive skill 3-6 weeks afterward.

S2S predictability can also be enhanced by land surface responses to atmospheric anomalies and by ocean dynamics and feedbacks such as Arctic sea ice, currents, and eddies

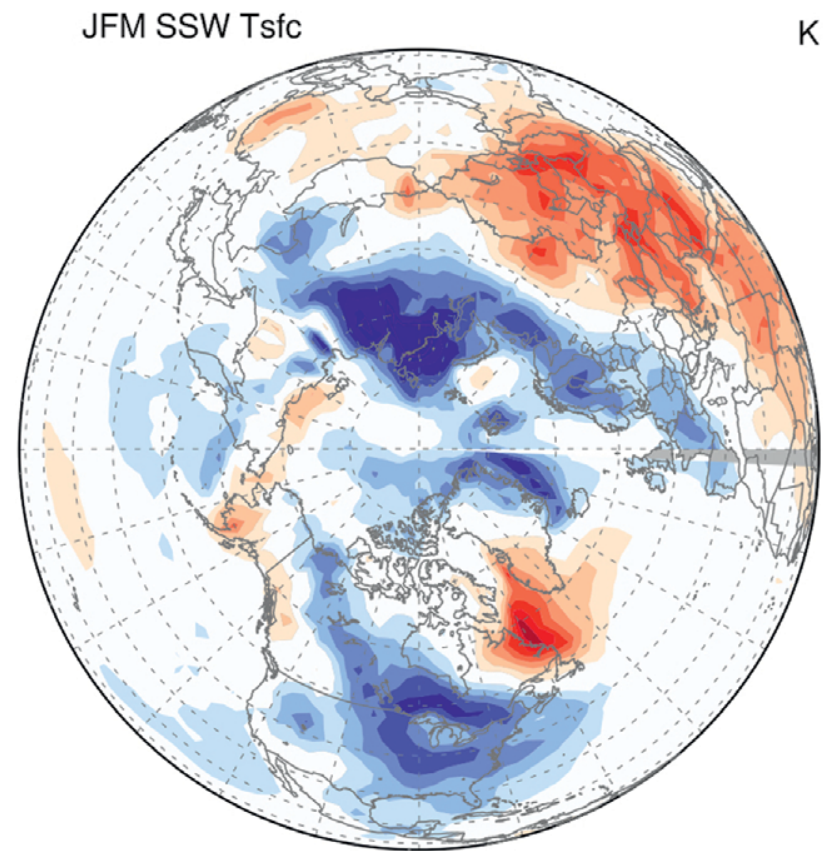

K JFM no-SSW Tsfc

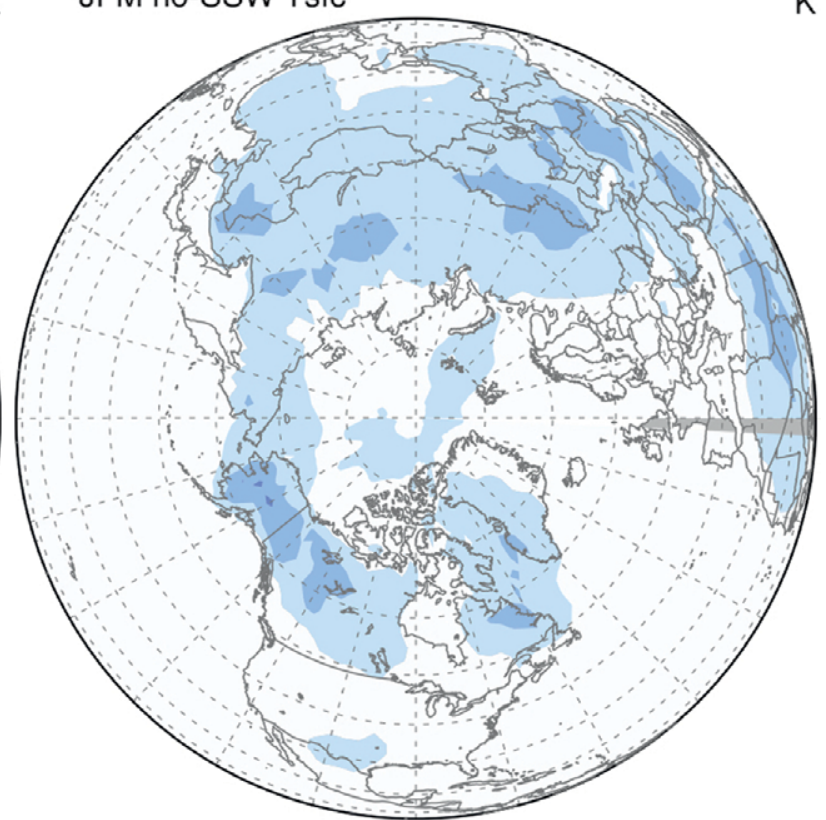

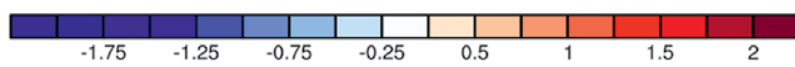

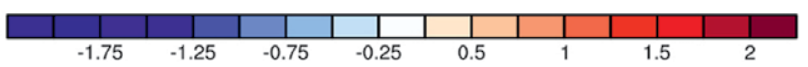




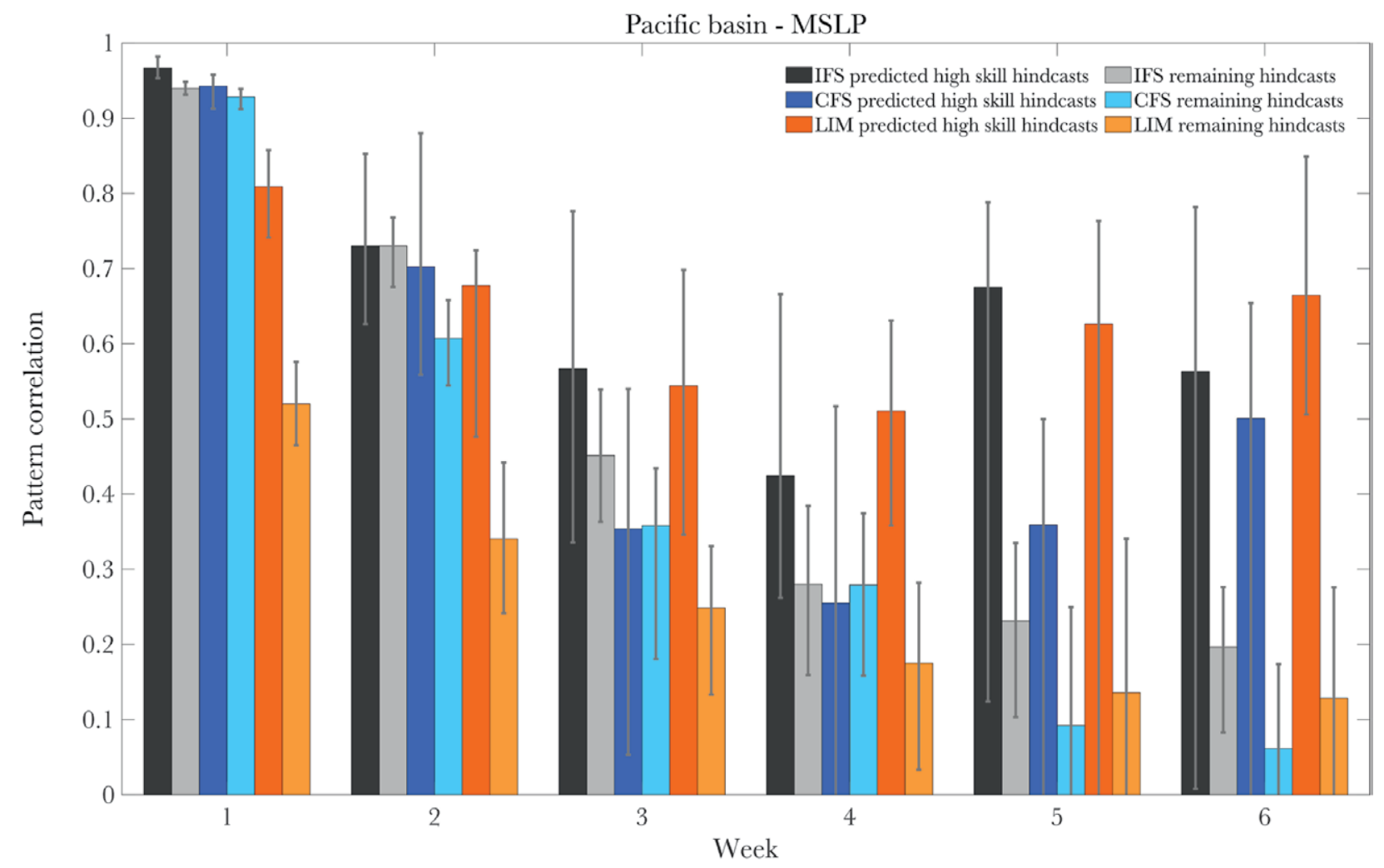

along ocean fronts. The Atlantic meridional overturning circulation, meanwhile, evolves on multidecadal time scales and may provide S2D predictability if it can be adequately simulated. Also on S2D time scales, volcanic eruptions can enhance predictability, as can anthropogenic aerosols.

\section{Building the forecast tools}

Tools are being developed to predict the skill of S2S/S2D forecasts during windows of opportunity. For example, predictability can be well approximated by relatively simple linear dynamics and a residual random "noise." Already, probabilistic forecasts over North America are based solely on the climatological trend and statistical relationships to the state of the MJO and ENSO, with some regions of skill at approximately 4-weeks lead time. Also, an experimental prediction framework is being developed to predict weekly atmospheric river frequency based on hindcast and forecast information, with conditional useful skill 1-3 weeks ahead.

Some studies report that S2S climate models can more skillfully predict events with greater anomalies than events closer to the mean, suggesting uses for outlooks for extreme events.
* The "linear inverse model," or LIM, is an empirical forecast model. Here it is applied to identify mean sea level pressure (MSLP) winter forecasts of opportunity in the Pacific basin for up to 6 weeks lead time. The LIM technique allows identification of high skill cases or forecasts of opportunity ahead of time. Such higher skill depends upon the similarity of the forecast initialization to patterns leading to the most rapidly amplifying anomalies within the LIM. The figure shows median hindcast skill (1999-2010) of wintertime Pacific basin MSLP weekly-averaged anomalies predicted by operational NCEP CFS and ECMWF IFS models and by the LIM model, for forecast leads ranging from 1 to 6 weeks. For each forecast system, two categories are shown: one where the LIM expects skill will lie in the upper $10 \%$ of all hindcasts (darker bars) and a second consisting of the remaining $90 \%$ of hindcasts (lighter bars). Whiskers denote uncertainty due to the small hindcast sample size (based on bootstrap confidence intervals). Skill is measured by the pattern correlation of the forecast MSLP anomaly with its verification in the region $20^{\circ}-60^{\circ} \mathrm{N}$, $120^{\circ} \mathrm{E}-120^{\circ} \mathrm{W}$. 
wind, etc., are issued with 2-week lead times. However, building tools on windows of opportunity presents cultural challenges. People are accustomed to forecasts on time, all the time. Adapting to probabilistic forecasts with information only in certain conditions requires a significant mindset shift-including for the forecasters.

Building tools for forecasts of opportunity requires, among other things, a large enough sample of past events to build statistically sound relationships. Yet forecasts of opportunity automatically decrease the sample size: only a subset of days-particularly for rare events-is considered as training data.

Research could point to new predictability sources beyond, say, ENSO, and help develop new opportunity-based forecasts. Applying a window of opportunity approach to examining observational data and forecast-system output could also reveal relationships and skill when and where none was otherwise apparent. There is also a need for evaluation diagnostics and metrics for such forecasts.

Improved skill of dynamical models is to be expected and purposefully sought after. For example, we are still trying to understand, simulate, and exploit ENSO variability better. It is logical to build opportunity-based forecasts considering the processes that we understand to enhance forecast skill and improve dynamical prediction. However, empirical models or dynamical models based on forecast output models (i.e., hybrid models) already show promise and may continue to be a synergetic, innovative way forward for exploring predictability-until underpinning mechanisms can be more fully understood and better simulated by dynamical forecast systems.

Disclaimer: The views expressed here are solely those of the authors and not of any U.S. federal government or state government agencies.
BAMS: What would you like readers to learn from this article?

Annarita Mariotti (NOAA): Not all forecast situations are alike. We need to better understand when and where there is opportunity for better forecasts, what modulates the degree of predictability, and how to exploit this knowledge most effectively toward useful forecasts.

BAMS: How did you become interested in the topic of this article?
AM: In my own research, I have studied how processes such as ENSO, the North Atlantic Oscillation. and the Atlantic multidecadal oscillation affect climate conditions differently depending on the timing and the location. Through the work of the NOAA S2S Prediction Task Force, which I helped to organize, it has become increasingly clear to me that the predictability of phenomena such as atmospheric rivers, heat waves, and other extremes is modulated by initial conditions and underlying climate phenomena, and that this knowledge can help inform improved approaches to predictions.

BAMS: What surprises/surprised you the most about the work you document in this article?
AM: When I first toyed with the idea of writing this article and started mentioning it around, I was frankly surprised that people thought the effort was useful and timely. There are decades of literature on processes like ENSO and the MJO, their inherent predictability, and their impacts, and also plenty of literature on prediction science and practice. Despite this, colleagues suggested that emphasizing forecast opportunities would be useful. 


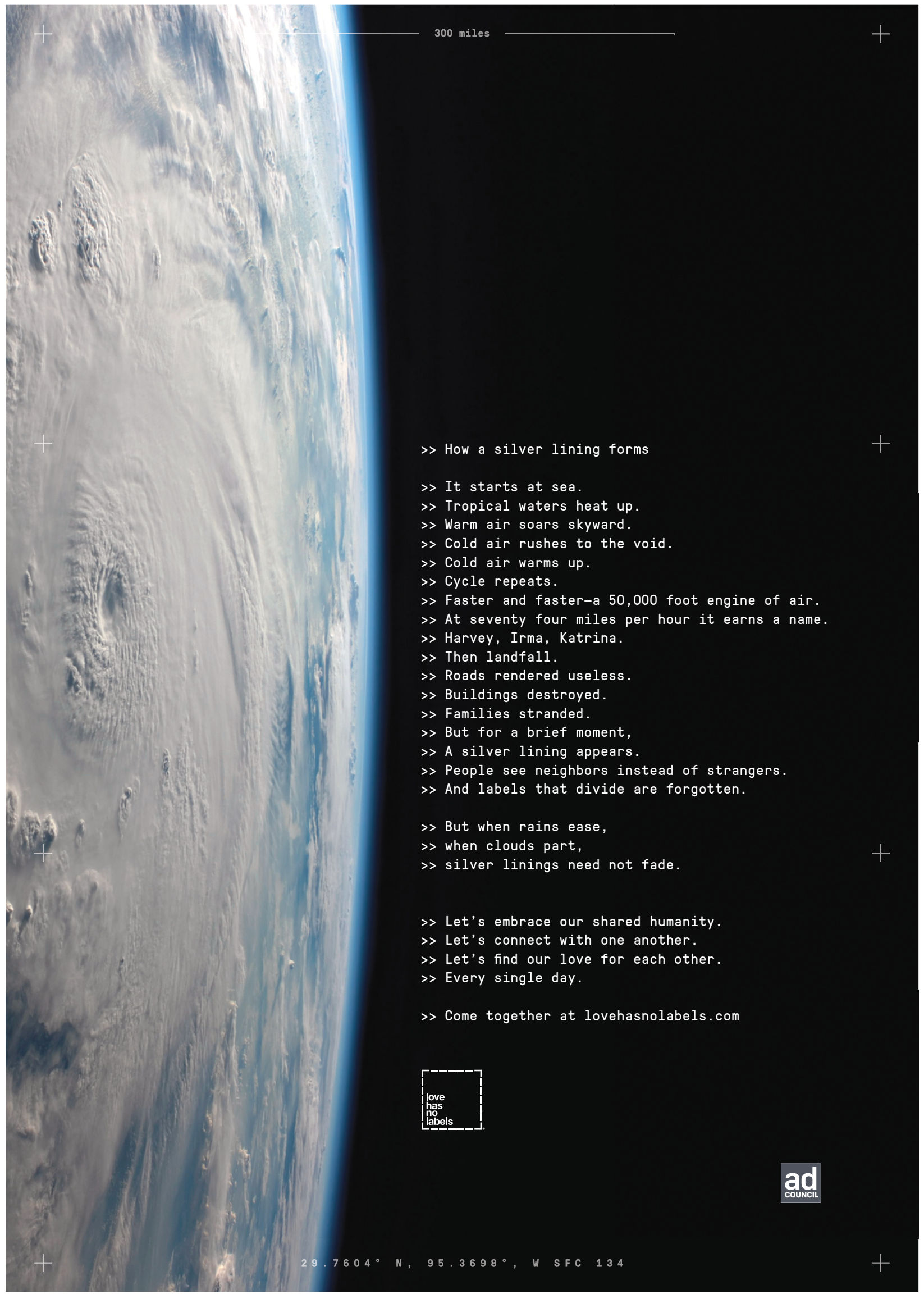

